\title{
Management of endometrial polyps in infertile women: A mini-review
}

\author{
Byung Chul Jee ${ }^{1,2}$, Hye Gyeong Jeong ${ }^{1,2}$ \\ 'Department of Obstetrics and Gynecology, Seoul National University Bundang Hospital, Seongnam; ${ }^{2}$ Department of Obstetrics and Gynecology, Seoul \\ National University College of Medicine, Seoul, Korea
}

Considerable disagreement exists regarding whether endometrial polyps should be removed before attempting natural pregnancy and before pregnancy via intrauterine insemination (IUI) or in vitro fertilization (IVF). Through a literature review, we obtained information on the impact of endometrial polyps and polypectomy on fertility outcomes. Several observational studies have suggested that women with unexplained infertility may benefit from endometrial polypectomy for a future natural pregnancy. A few studies reported benefits from endometrial polypectomy in infertile women who plan to undergo IUI. However, no strong evidence supports polypectomy as a way to improve the pregnancy rate in infertile women who plan to undergo IVF or polypectomy during controlled ovarian stimulation for IVF. Although no studies have defined criteria for the polyp size that should be removed in infertile women, clinicians should be aware that small endometrial polyps $(<10 \mathrm{~mm})$ sometimes regress spontaneously. Endometrial polypectomy is currently justified in patients with repeated IVF failure, but more studies are needed to verify that endometrial polypectomy itself will eventually increase the pregnancy rate. Although several mechanisms by which endometrial polyps exert a negative effect on fertility have emerged, there is no consensus about the proper management of endometrial polyps in infertile women. Therefore, the management of endometrial polyps should be individualized depending on the patient's situation and clinician's preference.

Keywords: Endometrial polyp; In vitro fertilization; Infertility; Intrauterine insemination; Polypectomy; Pregnancy

\section{Introduction}

Endometrial polyps are commonly seen in infertile women [1]. However, there is considerable disagreement regarding whether endometrial polyps should be removed before attempting natural pregnancy and pregnancy via intrauterine insemination (IUI) or in vitro fertilization (IVF) [2-4]. From a clinical point of view, the following questions are important: (1) should endometrial polyps be removed in infertile women who are attempting natural pregnancy and who are planning to undergo IUI or IVF? and (2) is there a standard for the size of endometrial polyps that should be removed?

Received: October 7, 2020 · Revised: March 6, 2021 · Accepted: April 9, 2021 Corresponding author: Hye Gyeong Jeong

Department of Obstetrics and Gynecology, Seoul National University Bundang Hospital, 82 Gumi-ro 173beon-gil, Bundang-gu, Seongnam 13620, Korea Tel: +82-31-787-6244 Fax: +82-31-787-4054 E-mail: psyche622@naver.com

This is an Open Access article distributed under the terms of the Creative Commons Attribution Non-Commercial License (http://creativecommons.org/licenses/by-nc/4.0/) which permits unrestricted non-commercial use, distribution, and reproduction in any medium, provided the original work is properly cited.
In this mini-review, we provide an overview of the impact of endometrial polyps on fertility and the impact of endometrial polypectomy on natural pregnancy and pregnancy via IUI or IVF. We also suggest the most appropriate approach to the management of endometrial polyps in infertile women.

\section{Endometrial polyps in infertile women who are attempting natural pregnancy}

Varasteh et al. [5] reported a pregnancy rate of 78.3\% after endometrial polypectomy in infertile women compared with $42.1 \%$ in those with a normal uterine cavity. Spiewankiewicz et al. [6] reported a pregnancy rate of $76 \%$ during 12 months after endometrial polypectomy in infertile women, and Shokeir et al. [7] reported a 50\% pregnancy rate after endometrial polypectomy. These observational studies suggest that women with unexplained infertility may benefit from endometrial polypectomy for a future natural pregnancy.

The location of endometrial polyps may influence the pregnancy 
rate. The pregnancy rate after endometrial polypectomy was highest for polyps located at the utero-tubal junction (57.4\%), followed by $40.3 \%$ for multiple polyps, $28.5 \%$ for posterior uterine wall polyps, $18.8 \%$ for lateral uterine wall polyps, and $14.8 \%$ for anterior uterine wall polyps [8]. However, endometrial polypectomy may improve fertility irrespective of the size or number of polyps. No difference was found in the pregnancy or miscarriage rate after hysteroscopic polypectomy for small polyps ( $\leq 10 \mathrm{~mm}$ ) compared to larger or multiple polyps [9].

\section{Endometrial polyps in infertile women undergoing IUI}

Perez-Medina et al. [10] performed a randomized controlled trial in women undergoing IUI. The inclusion criteria were women with at least 24 months of inability to conceive and a sonographic diagnosis of endometrial polyps, who were candidates for IUI. They compared the cumulative clinical pregnancy rate after four IUI cycles between women who underwent hysteroscopic polypectomy versus those who underwent diagnostic hysteroscopy and a polyp biopsy only. The size of the endometrial polyps was similar in both groups (mean, $16 \mathrm{~mm}$; range, 3-24 mm). The first IUI was planned for three cycles after hysteroscopy in both groups. They reported a significantly higher cumulative pregnancy rate in the patients who underwent hysteroscopic polypectomy than in those who underwent diagnostic hysteroscopy and polyp biopsy (51.4\% vs. $25.4 \%$; relative risk [RR], 2.1; $95 \%$ confidence interval [Cl], 1.5-2.9). In their report, endometrial polyps $\leq 10 \mathrm{~mm}$ accounted for $56 \%$ and $61 \%$ in the two groups, respectively. Unfortunately, the pregnancy rate was not presented according to the size of the endometrial polyps.

In women undergoing IUI, Kalampokas et al. [11] retrospectively compared the cumulative clinical pregnancy rate after three IUI cycles between a group of patients who underwent hysteroscopic polypectomy versus a group with remaining. The size of the endometrial polyps was similar in both groups (mean, $13.6 \mathrm{~mm}$ vs. $12.0 \mathrm{~mm}$ ). Endometrial polyps $\leq 10 \mathrm{~mm}$ accounted for $54.6 \%$ and $58.8 \%$ in the two groups, respectively, and endometrial polyps $>20 \mathrm{~mm}$ accounted for $24.4 \%$ and $20 \%$ in the two groups, respectively. They reported a significantly higher cumulative pregnancy rate in the group with hysteroscopic polypectomy ( $40.7 \%$ vs. $22.3 \%$ ).

\section{Endometrial polyps in infertile women undergoing IVF}

Lass et al. [12] retrospectively compared IVF cycle outcomes between 49 women who underwent fresh embryo transfer (ET) without polypectomy versus 34 women who received cryopreservation of all embryos and underwent polypectomy followed by frozen ET 3 months. The endometrial polyps were less than $20 \mathrm{~mm}$ and were diagnosed by transvaginal ultrasonography. The pregnancy rate was similar ( $22.4 \%$ vs. $23.4 \%)$, but the miscarriage rate was higher in the group with fresh ET without polypectomy ( $27.3 \%$ vs. $10.7 \%, p=0.08)$. The authors concluded that endometrial polyps $<20 \mathrm{~mm}$ did not decrease the pregnancy rate, but there was a trend toward increased pregnancy loss. However, a histological diagnosis of the endometrial polyp was made in only $58.3 \%$ of the polypectomy group.

Isikoglu et al. [13] retrospectively compared intracytoplasmic sperm injection (ICSI) cycle outcomes among three groups: (1) 15 women with polyps remaining, (2) 40 women who underwent hysteroscopic polypectomy, and (3) 956 women with no polyps. All endometrial polyps were less than $15 \mathrm{~mm}$ and were discovered during controlled ovarian stimulation (COS). The clinical pregnancy rate and implantation rate were similar among the three groups. The authors concluded that endometrial polyps discovered during COS did not negatively affect pregnancy and implantation outcomes in ICSI cycles.

Furthermore, Check et al. [14] confirmed a similar clinical pregnancy rate and miscarriage rate after IVF cycles among three groups: (1) women who underwent polypectomy, (2) women with polyps remaining, and (3) women without polyps. Tiras et al. [15] retrospectively compared ICSI cycle outcomes among four groups: (1) 47 women diagnosed with endometrial polyps before the ICSI cycle who underwent hysteroscopic polypectomy, (2) 47 matched controls with no polyps, (3) 128 women diagnosed with endometrial polyps during COS, and (4) 128 matched controls with no polyps. The size of polyps was only identified in 98 women in group 3, and ranged from $4 \mathrm{~mm}$ to $14 \mathrm{~mm}$. The clinical pregnancy rate (29.8\% vs. $38.3 \%)$ and live birth rate ( $25.5 \%$ vs. $31.9 \%$ ) were similar between groups 1 and 2 , and the clinical pregnancy rate $(45.3 \%$ vs. $46.9 \%)$ and live birth rate $(40.6 \%$ vs. $39.8 \%)$ were also similar between groups 3 and 4 . From their study, it is unknown whether hysteroscopic polypectomy increases the pregnancy rate in ICSI cycles, because the control group was not composed of women with polyps remaining. However, their study suggests that endometrial polyps diagnosed during COS do not decrease the pregnancy rate in ICSI cycles.

Elias et al. [16] retrospectively compared IVF cycle outcomes between 60 women with endometrial polyps diagnosed during COS versus 2,933 women without polyps. The clinical pregnancy, spontaneous miscarriage, and live birth rates were similar between the two groups. However, the biochemical pregnancy rate was significantly higher in the polyp group than in the non-polyp group (18.3\% vs. 9.6\%). The authors concluded that newly diagnosed endometrial polyps during $\operatorname{COS}$ were associated with increased biochemical pregnancy rate, but ultimately did not adversely impact the clinical pregnancy or live birth rates after fresh IVF. 
If endometrial polyps are noticed during COS for a fresh IVF-ET cycle, two options are possible: (1) proceeding with fresh ET without polypectomy and (2) freezing all embryos and performing polypectomy with frozen ET later. However, a third option may be possible (i.e., polypectomy during $\mathrm{COS}$ and proceeding with fresh ET). Although the number of women was small in two studies, reasonable pregnancy rates (4/9 and 3/6) were obtained when hysteroscopic polypectomy was performed during COS followed by fresh $\mathrm{ET}[17,18]$.

The effects of the so-called "without cycle cancellation" policy (i.e., polypectomy during COS followed by fresh ET) were also reported in a subsequent non-randomized study [19]. The authors compared the IVF cycle outcomes between a study group (polypectomy during COS followed by fresh ET without cycle cancellation) versus a control group (without polypectomy and proceeding with fresh ET). Endometrial polyps were all diagnosed incidentally during $\operatorname{COS}$ for fresh IVF cycles (size range, 5-20 mm). Endometrial polyps were all confirmed pathologically in the study group. The average diameter of the polyps in the study group was higher than that of the polyps in the control group $(10.0 \pm 4.0 \mathrm{~mm}$ vs. $13.3 \pm 5.7 \mathrm{~mm}, p=0.001)$. The two groups were similar in terms of the clinical pregnancy rate, implantation rate, and live birth rate. In the study group, the interval between polypectomy and ET was 3-17 days, and this was the only significant predictor of the live birth rate. No pregnancies were achieved in women with an interval $<5$ days. In the subgroup with an interval of 5-9 days, the pregnancy rate was $39.5 \%$, and in the subgroup with an interval of $>10$ days, the pregnancy rate was $40 \%$. The authors suggested that polypectomy is unnecessary during fresh IVF-ET cycles because polypectomy did not improve the cycle outcomes.

The study by Ghaffari et al. [19] may provide insights into whether polypectomy should be performed in fresh IVF-ET cycles. Based on the aforementioned studies, endometrial polyps $<20 \mathrm{~mm}$ appear to have no impact on IVF outcomes. Although the real impact of endometrial polyps on fertility is uncertain, most of the aforementioned studies favor expectant management of endometrial polyps diagnosed in women planning to undergo IVF. If polyp removal is unnecessary in women planning to undergo IVF, we can reduce the number of unnecessary surgical procedures in many cases. In a prospective study of 1,000 women undergoing hysteroscopic evaluation of the uterine cavity prior to IVF, the prevalence of endometrial polyps was found to be $32 \%$ [1].

\section{Size criteria for endometrial polyps that should be removed}

No study has established criteria for the size of polyps that should be removed or could be observed in infertile women. However, small endometrial polyps $(<10 \mathrm{~mm}$ ) sometimes regress spontaneously [20]. Therefore, the American Association of Gynecologic Laparoscopists guideline states that management of endometrial polyps may be conservative, with up to $25 \%$ of polyps regressing, particularly if they are less than $10 \mathrm{~mm}$ in size [21].

\section{Messages from other systematic reviews}

In two systematic reviews and 1 practice guideline, removal of endometrial polyps was recommended if they are identified in infertile women [2,3,21]. However, Afifi et al. [3] and Pereira et al. [4] advocated that the management of endometrial polyps observed during COS for IVF should be individualized considering the number of embryos formed, the woman's previous reproductive history, and the individual clinic's success with frozen ET.

In a recent systematic review, Zhang et al. [22] reviewed eight studies regarding hysteroscopic polypectomy and assisted reproductive technology outcomes compared with no treatment. They concluded that hysteroscopic polypectomy was associated with an increased rate of clinical pregnancy in patients who underwent IUI, but no clear benefit was observed for clinical pregnancy, live birth, miscarriage, or implantation rates in patients who underwent IVF.

\section{Negative impact of endometrial polyps on fertility}

If endometrial polypectomy is helpful in women trying natural pregnancy or IUI cycles, why are they not helpful in women trying IVF cycles? Why do endometrial polyps not have a negative effect on IVF pregnancies? It is believed that the high implantation potential of IVF can overcome the negative impact of endometrial polyps.

The mechanism by which endometrial polyps may interfere with implantation is not clear. Endometrial polyps may interfere with embryo implantation because endometrial polyps are space-occupying lesions. In a uterine flushing study, an increased concentration of glycodelin in the proliferative and preovulatory phase (cycle day 5-14) was found in women with endometrial polyps [23]. It is known that endometrial glycodelin levels are absent or low during the follicular phase and peri-ovulatory period, but increase during the implantation period [24]. Although glycodelin levels were not measured precisely during the peri-ovulatory period in the uterine flushing study, the authors speculated that endometrial receptivity might be altered by increased glycodelin production in the uterine cavity of patients with polyps.

It has been reported that the activated mast cell number increases more than seven-fold throughout the menstrual cycle in the endometrial cavity in women with endometrial polyps [25]. Mast cells 
play a key role in inflammatory and allergic processes, and when activated, they release several substances such as histamine, prostaglandin, leukotrienes, and cytokines. The levels of cytokines, as well as matrix metalloproteinase- 2 and matrix metalloproteinase- 9 , were also found to be elevated in the uterine cavity in women with endometrial polyps [26]. Thus, endometrial polyps may induce local inflammatory changes, which can interfere with normal embryo implantation. Moreover, endometrial polyps may affect endometrial receptivity via decreased mRNA levels of HOXA10 and HOXA11, which may impair normal embryo implantation [27].

\section{Endometrial polyps in women with repeated IVF failure}

In patients with repeated IVF failure (RIF), the principle is to identify intrauterine lesions and correct any abnormalities. Demirol and Gurgan [28] reported that intrauterine abnormalities were found in $26 \%$ of cases via repeated examinations, even though initial hysterosalpingography and diagnostic hysteroscopy were normal. They divided 421 RIF patients into two groups: 210 patients underwent hysteroscopy and 211 patients did not. In patients who underwent hysteroscopy, 56 patients were found to have abnormalities (such as endometrial hyperplasia, polyps, endometritis, synechia, or myoma) that were surgically corrected. The pregnancy rate of the surgically corrected group was $32.5 \%$, which was significantly higher than the rate of $21.6 \%$ in the non-hysteroscopy group. Interestingly, the pregnancy rate of those who showed normal findings after hysteroscopy was also high (30.4\%). This finding supports the idea that the hysteroscopy alone can increase the pregnancy rate.

According to a systematic review, the incidence of abnormal hysteroscopic findings in patients with RIF varied between $25 \%$ and $50 \%$, and pooling data from randomized studies showed that hysteroscopy significantly increased the clinical pregnancy rate in the subsequent IVF cycle (pooled RR, 1.57; 95\% Cl, 1.29-1.92) [29]. It is not certain whether sole endometrial polypectomy will definitely increase pregnancy rate in patients with RIF, but polypectomy is at least justified in RIF patients.

\section{Conclusion}

Based on the literature review presented above, the following recommendations can be made. (1) Women with unexplained infertility may benefit from endometrial polypectomy for a future natural pregnancy. However, the level of evidence is low. (2) Infertile women who plan to undergo IUI may benefit from endometrial polypectomy. However, this is based on only one prospective randomized study. (3) In infertile women who plan to undergo IVF, there is no strong evidence to support polypectomy (or polypectomy during COS for IVF) in order to enhance the pregnancy rate. (4) No study has yet determined criteria for the size of polyps that should be removed or could be observed in infertile women. However, clinicians should be aware that small endometrial polyps ( $<10 \mathrm{~mm}$ ) sometimes regress spontaneously. (5) Endometrial polypectomy is justified in patients with RIF, but more studies are needed to verify that endometrial polypectomy itself will eventually increase the pregnancy rate. (6) There remains no consensus about the proper management of endometrial polyps in infertile women. Therefore, the management of endometrial polyps should be individualized depending on the patient's situation and clinician's preference.

\section{Conflict of interest}

Byung Chul Jee has been the editor-in-chief of Clinical and Experimental Reproductive Medicine since 2018; however, he was not involved in the peer reviewer selection, evaluation, or decision process of this article. No other potential conflict of interest relevant to this article was reported.

\section{ORCID}

Byung Chul Jee https://orcid.org/0000-0003-2289-6090 Hye Gyeong Jeong https://orcid.org/0000-0002-8608-748X

\section{Author contributions}

Conceptualization, Data curation, Formal analysis, Methodology, Writing-original draft, Writing-review \& editing: all authors.

\section{References}

1. Hinckley MD, Milki AA. 1000 office-based hysteroscopies prior to in vitro fertilization: feasibility and findings. JSLS 2004;8:103-7.

2. Taylor E, Gomel V. The uterus and fertility. Fertil Steril 2008;89:116.

3. Afifi K, Anand S, Nallapeta S, Gelbaya TA. Management of endometrial polyps in subfertile women: a systematic review. Eur J Obstet Gynecol Reprod Biol 2010;151:117-21.

4. Pereira N, Petrini AC, Lekovich JP, Elias RT, Spandorfer SD. Surgical management of endometrial polyps in infertile women: a comprehensive review. Surg Res Pract 2015;2015:914390.

5. Varasteh NN, Neuwirth RS, Levin B, Keltz MD. Pregnancy rates after hysteroscopic polypectomy and myomectomy in infertile women. Obstet Gynecol 1999;94:168-71.

6. Spiewankiewicz B, Stelmachow J, Sawicki W, Cendrowski K, Wy- 
pych P, Swiderska K. The effectiveness of hysteroscopic polypectomy in cases of female infertility. Clin Exp Obstet Gynecol 2003; 30:23-5.

7. Shokeir TA, Shalan HM, El-Shafei MM. Significance of endometrial polyps detected hysteroscopically in eumenorrheic infertile women. J Obstet Gynaecol Res 2004;30:84-9.

8. Yanaihara A, Yorimitsu T, Motoyama H, Iwasaki S, Kawamura T. Location of endometrial polyp and pregnancy rate in infertility patients. Fertil Steril 2008;90:180-2.

9. Stamatellos I, Apostolides A, Stamatopoulos P, Bontis J. Pregnancy rates after hysteroscopic polypectomy depending on the size or number of the polyps. Arch Gynecol Obstet 2008;277:395-9.

10. Perez-Medina T, Bajo-Arenas J, Salazar F, Redondo T, Sanfrutos L, Alvarez $\mathrm{P}$, et al. Endometrial polyps and their implication in the pregnancy rates of patients undergoing intrauterine insemination: a prospective, randomized study. Hum Reprod 2005;20:1632 -5 .

11. Kalampokas T, Tzanakaki D, Konidaris S, lavazzo C, Kalampokas E, Gregoriou O. Endometrial polyps and their relationship in the pregnancy rates of patients undergoing intrauterine insemination. Clin Exp Obstet Gynecol 2012;39:299-302.

12. Lass A, Williams G, Abusheikha N, Brinsden P. The effect of endometrial polyps on outcomes of in vitro fertilization (IVF) cycles. J Assist Reprod Genet 1999;16:410-5.

13. Isikoglu M, Berkkanoglu M, Senturk Z, Coetzee K, Ozgur K. Endometrial polyps smaller than $1.5 \mathrm{~cm}$ do not affect ICSI outcome. Reprod Biomed Online 2006;12:199-204.

14. Check JH, Bostick-Smith CA, Choe JK, Amui J, Brasile D. Matched controlled study to evaluate the effect of endometrial polyps on pregnancy and implantation rates following in vitro fertilization-embryo transfer (IVF-ET). Clin Exp Obstet Gynecol 2011;38: 206-8.

15. Tiras B, Korucuoglu U, Polat M, Zeyneloglu HB, Saltik A, Yarali H. Management of endometrial polyps diagnosed before or during ICSI cycles. Reprod Biomed Online 2012;24:123-8.

16. Elias RT, Pereira N, Karipcin FS, Rosenwaks Z, Spandorfer SD. Impact of newly diagnosed endometrial polyps during controlled ovarian hyperstimulation on in vitro fertilization outcomes. J Minim Invasive Gynecol 2015;22:590-4.

17. Batioglu S, Kaymak O. Does hysteroscopic polypectomy without cycle cancellation affect IVF? Reprod Biomed Online 2005;10: 767-9.
18. Madani T, Ghaffari F, Kiani K, Hosseini F. Hysteroscopic polypectomy without cycle cancellation in IVF cycles. Reprod Biomed Online 2009;18:412-5.

19. Ghaffari F, Arabipoor A, Bagheri Lankarani N, Hosseini F, Bahmanabadi A. Hysteroscopic polypectomy without cycle cancellation in IVF/ICSI cycles: a cross-sectional study. Eur J Obstet Gynecol Reprod Biol 2016;205:37-42.

20. DeWaay DJ, Syrop CH, Nygaard IE, Davis WA, Van Voorhis BJ. Natural history of uterine polyps and leiomyomata. Obstet Gynecol 2002;100:3-7.

21. American Association of Gynecologic Laparoscopists. AAGL practice report: practice guidelines for the diagnosis and management of endometrial polyps. J Minim Invasive Gynecol 2012;19: 3-10.

22. Zhang H, He X, Tian W, Song X, Zhang H. Hysteroscopic resection of endometrial polyps and assisted reproductive technology pregnancy outcomes compared with no treatment: a systematic review. J Minim Invasive Gynecol 2019;26:618-27.

23. Richlin SS, Ramachandran S, Shanti A, Murphy AA, Parthasarathy S. Glycodelin levels in uterine flushings and in plasma of patients with leiomyomas and polyps: implications for implantation. Hum Reprod 2002;17:2742-7.

24. Li TC, Ling E, Dalton C, Bolton AE, Cooke ID. Concentration of endometrial protein PP14 in uterine flushings throughout the menstrual cycle in normal, fertile women. Br J Obstet Gynaecol 1993; 100:460-4.

25. Al-Jefout M, Black K, Schulke L, Berbic M, Luscombe G, Tokushige $\mathrm{N}$, et al. Novel finding of high density of activated mast cells in endometrial polyps. Fertil Steril 2009;92:1104-6.

26. Inagaki N, Ung L, Otani T, Wilkinson D, Lopata A. Uterine cavity matrix metalloproteinases and cytokines in patients with leiomyoma, adenomyosis or endometrial polyp. Eur J Obstet Gynecol Reprod Biol 2003;111:197-203.

27. Rackow BW, Jorgensen E, Taylor HS. Endometrial polyps affect uterine receptivity. Fertil Steril 2011;95:2690-2.

28. Demirol A, Gurgan T. Effect of treatment of intrauterine pathologies with office hysteroscopy in patients with recurrent IVF failure. Reprod Biomed Online 2004;8:590-4.

29. Makrakis E, Pantos K. The outcomes of hysteroscopy in women with implantation failures after in-vitro fertilization: findings and effect on subsequent pregnancy rates. Curr Opin Obstet Gynecol 2010:22:339-43. 